\title{
PRODUCT INTEGRAL APPROXIMATIONS OF SOLUTIONS TO LINEAR OPERATOR EQUATIONS ${ }^{1}$
}

\author{
ROBERT H. MARTIN, JR.
}

\begin{abstract}
In the paper we develop a class of iterative methods for approximating solutions of linear operator equations in a Banach space. The main techniques involve a product integral representation of solutions to linear Stieltjes integral equations, a variation of parameters formula, and the asymptotic convergence of solutions to the homogeneous integral equation.
\end{abstract}

Let $E$ be a real or complex Banach space and let $|\cdot|$ denote the norm on $E$. Denote by $\mathscr{L}(E)$ the Banach algebra of all bounded linear transformations from $E$ into $E$ with the norm $\|\cdot\|$ on $\mathscr{L}(E)$ defined by $\|A\|=\sup \{|A x|:|x| \leqq 1\}$. In [1] F. E. Browder and W. V. Petryshyn show that if $T$ is a member of $\mathscr{L}(E)$ which is asymptotically convergent (i.e., $\lim _{n \rightarrow \infty} T^{n} x$ exists for each $x$ in $\left.E\right), I$ is the identity of $\mathscr{L}(E)$, and $z$ is in the range of $I-T$, then the iterative process

$$
x_{n+1}=T x_{n}+z \quad(n=0,1,2, \cdots)
$$

converges to a solution $x^{*}$ of the equation

$$
x-T x=z,
$$

for any initial approximation $x_{0}$ in $E$. In [3] and [4] W. G. Dotson extends this result to a more general class of iterative processes by using mean ergodic theorems (see also D. G. DeFigueiredo and L. A. Karlovitz [2]). It is the purpose of this note to extend the results of [1] by using the theory of product integrals and Stieltjes integral equations developed by J. S. Mac Nerney in [6] and [7].

Throughout this paper we assume that $S$ is a subset of $[0, \infty)$ such that $0 \in S$ and $\sup \{t: t \in S\}=\infty$. Also, $g$ is an increasing function from $S$ into $[0, \infty)$ such that $g(0)=0$ and $\sup \{g(t): t \in S\}=\infty$. For notational convenience, let $\Delta=\{(s, t) \in S \times S: s \leqq t\}$. If $(s, t) \in \Delta$ and $u=\left(u_{k}\right)_{0}^{n}$ is a

Received by the editors May 4, 1972.

AMS (MOS) subject classifications (1970). Primary 47A50; Secondary 45A05.

Key words and phrases. Linear operator equations, product integral, Stieltjes integral equation, asymptotic convergence.

${ }^{1}$ This work was supported in part by the U.S. Army Research Office, Durham, North Carolina.

(c) American Mathematical Society 1973 
finite sequence in $S$, then $u$ is said to be a subdivision of $(s, t)$ if $u_{0}=s$, $u_{n}=t$, and $u_{k-1} \leqq u_{k}$ for $k=1, \cdots, n$. If $f$ is a function from $S$ into $E$ and $(s, t)$ is in $\Delta$, then

$$
(R) \int_{s}^{t} f(\cdot) d g \text { and }(L) \int_{s}^{t} f(\cdot) d g
$$

denote the limits, in the sense of refinements of subdivisions, of members of $E$ of the form

$$
\sum_{1}^{n} f\left(u_{k}\right)\left[g\left(u_{k}\right)-g\left(u_{k-1}\right)\right] \text { and } \sum_{1}^{n} f\left(u_{k-1}\right)\left[g\left(u_{k}\right)-g\left(u_{k-1}\right)\right]
$$

respectively, where $\left(u_{k}\right)_{0}^{n}$ is a subdivision of $(s, t)$. If $\left\{F_{k}: k=1, \cdots, n\right\}$ is a family of functions from $E$ into $E$ and $x$ is in $E$, then $\prod_{1}^{n}\left[F_{k}\right] x$ denotes the left to right (functional composition) product $F_{1} \cdot F_{2} \cdots F_{n} x$. If $F$ is a function from $E$ into $E, x$ is in $E$, and $(s, t) \in \Delta$, then ${ }_{s} \Pi^{t}[I+d g F] x$ denotes the limit, in the sense of refinements of subdivisions, of members of $E$ of the form $\prod_{1}^{n}\left[I+\left(g\left(u_{k}\right)-g\left(u_{k-1}\right)\right) F\right] x$ where $\left(u_{k}\right)_{0}^{n}$ is a subdivision of $(s, t)$.

Now let $A$ be a member of $\mathscr{L}(E)$ and for each $(s, t) \in \Delta$ define the member $W(s, t)$ of $\mathscr{L}(E)$ by

$$
W(s, t) x={ }_{s} \prod^{t}[I+d g A] x
$$

for each $x \in E$. It is shown in [6, Theorems 3.3 and 3.4] that $W$ is well defined, and for each $x$ in $E$ and $(s, t)$ in $\Delta$,

and

$$
W(s, t) x=x+(R) \int_{s}^{t} A W(\cdot, t) x d g
$$

$$
W(s, t) x=x+(L) \int_{s}^{t} W(s, \cdot) A x d g .
$$

Since $A[I+(g(u)-g(v)) A]=[I+(g(u)-g(v)) A] A$ for all $(u, v) \in \Delta$, it follows easily that $A W(s, t)=W(s, t) A$ for all $(s, t) \in \Delta$. Also, let $z$ be in $E$ and let $A+z$ denote the (affine) mapping $F$ from $E$ into $E$ defined by $F x=A x+z$. For each $(s, t) \in \Delta$ and $x \in E$ define

$$
M(s, t) x={ }_{s} \prod^{t}[I+d g(A+z)] x .
$$

It follows from the results of Mac Nerney [7, Theorem 1.1 and Corollary 2.1] (see, in particular, the remark on p. 637 of [7]) that $M$ is well defined and

$$
M(s, t) x=x+(R) \int_{s}^{t} A M(\cdot, t) x d g+(g(t)-g(s)) z
$$

for all $(s, t) \in \Delta$ and $x \in E$. 
The first lemma is crucial for our analysis and is essentially a variation of parameters formula for the solutions of (6). For further results of this type see D. L. Lovelady [5, Lemma 7] and J. A. Reneke [8].

Lemma 1. Suppose that $W$ is defined by (3) and $M$ is defined by (5). Then

$$
M(s, t) x=W(s, t) x+(R) \int_{s}^{t} W(\cdot, t) z d g
$$

for all $(s, t)$ in $\Delta$ and $x$ in $E$.

Indication of Proof. For each $(s, t) \in \Delta$ and $x \in E$ let $M^{\prime}(s, t) x$ be defined by the right side of (7). It is easy to see that $M^{\prime}(\cdot, t) x$ is of bounded variation on the $S$ interval $[0, t]$. Let

$$
P=x+(R) \int_{s}^{t} A M^{\prime}(\cdot, t) x d g+(g(t)-g(s)) z .
$$

If $\left(u_{k}\right)_{0}^{n}$ is a subdivision of $(s, t)$ and $\delta_{k} g=g\left(u_{k}\right)-g\left(u_{k-1}\right)$ for $k=1, \cdots, n$, then

$$
\begin{aligned}
P & \sim x+\sum_{1}^{n} A\left[W\left(u_{k}, t\right) x+(R) \int_{u_{k}}^{t} W(\cdot, t) z d g\right] \delta_{k} g+\sum_{1}^{n} z \delta_{k} g \\
& =x+\sum_{1}^{n} A W\left(u_{k}, t\right) x \delta_{k} g+\sum_{1}^{n}\left[z+A(R) \int_{u_{k}}^{t} W(\cdot, t) z d g\right] \delta_{k} g .
\end{aligned}
$$

Also, $z+A(R) \int_{u_{k}}^{t} W(\cdot, t) z d g=z+(R) \int_{u_{k}}^{t} A W(\cdot, t) z d g=W\left(u_{k}, t\right) z$ by (4); so

$$
\begin{aligned}
P & \sim x+\sum_{1}^{n} A W\left(u_{k}, t\right) x \delta_{k} g+\sum_{1}^{n} W\left(u_{k}, t\right) z \delta_{k} g \\
& \sim x+(R) \int_{s}^{t} A W(\cdot, t) x d g+(R) \int_{s}^{t} W(\cdot, t) z d g \\
& =W(s, t) x+(R) \int_{s}^{t} W(\cdot, t) z d g=M^{\prime}(s, t) x .
\end{aligned}
$$

Using the above estimates, it easily follows that

$$
M^{\prime}(s, t) x=x+(R) \int_{s}^{t} A M^{\prime}(\cdot, t) x d g+(g(t)-g(s)) z .
$$

Since the solution to (6) is unique by [7, Corollary 2.1], we have that $M^{\prime}=$ $M$ and the lemma is proved.

The function $W$ from $\Delta$ into $\mathscr{L}(E)$ defined by (3) is said to be asymptotically convergent if $\lim _{t \rightarrow \infty} W(0, t) x$ exists for each $x$ in $E$. Here and in the remainder of this paper, $\lim _{t \rightarrow \infty}$ denotes the limit as $t$ tends to infinity 
through values in $S$. Also, if $W$ is asymptotically convergent, let

$$
Q x=\lim _{t \rightarrow \infty} W(0, t) x
$$

for each $x$ in $E$.

LeMmA 2. Suppose that $W$ is asymptotically convergent and $Q$ is defined by (8). Then

(i) $Q$ is in $\mathscr{L}(E)$ and $Q^{2}=Q$;

(ii) $A Q=Q A=0$ and $W(s, t) Q=Q W(s, t)=Q$ for all $(s, t)$ in $\Delta$; and

(iii) the range of $Q$ is the null space of $A$.

Indication of Proof. Using (4) ${ }^{\prime}$ with $s=0$, we have that

$$
\lim _{t \rightarrow \infty}(L) \int_{0}^{t} W(0, \cdot) A x d g=Q x-x .
$$

Thus, since $\lim _{s \rightarrow \infty} W(0, s) A x=Q A x$ and $\lim _{s \rightarrow \infty} g(s)=\infty$, it is straightforward to show that $Q A x=\theta$ (where $\theta$ is the zero of $E$ ). Since $W(0, t) A=$ $A W(0, t)$ and $W(s, t) x=x$ if $A x=\theta$, part (ii) is immediate. Also, $Q$ is in $\mathscr{L}(E)$ by the uniform boundedness theorem and $Q^{2}=\lim _{t \rightarrow \infty} W(0, t) Q=$ $Q$ by (ii). Hence (i) is true. Finally, if $x$ is in $E$ and $A x=\theta$, then $W(0, t) x=$ $x$ for all $t$ in $S$ and we have that $Q x=x$. Part (iii) now follows easily and the proof is complete.

Letting $A=T-I$, we have that equation (2) becomes

$$
A x+z=\theta \text {, }
$$

where $\theta$ is the zero of $E$. With the approach used in this paper, it is more convenient to study the existence of solutions to (9) as opposed to (2).

Theorem 1. Suppose that $W$ is defined by (3), $M$ is defined by (5), and $W$ is asymptotically convergent. If $z$ is in the range of $A$ and $x_{0}$ is in $E$, then $x^{*}=\lim _{t \rightarrow \infty} M(0, t) x_{0}$ exists and $A x^{*}+z=\theta$.

Proof. Let $y \in E$ be such that $A y=z$. Using (7), (4), and the fact that $W(s, t) A=A W(s, t)$ we have that

$$
\begin{aligned}
M(0, t) x_{0} & =W(0, t) x_{0}+(R) \int_{0}^{t} W(\cdot, t) A y d g \\
& =W(0, t) x_{0}+(R) \int_{0}^{t} A W(\cdot, t) y d g \\
& =W(0, t) x_{0}+W(0, t) y-y .
\end{aligned}
$$

Hence $x^{*}=\lim _{t \rightarrow \infty} M(0, t) x_{0}=Q x_{0}+Q y-y$ where $Q$ is defined by (8). Thus $A x^{*}+z=A(-y)+z=\theta$ by (ii) of Lemma 2 . 
If $\left(x_{n}\right)_{1}^{\infty}$ is a sequence in $E$ which converges weakly to a member $x$ of $E$, we write $w$ - $\lim _{n \rightarrow \infty} x_{n}=x$. Also, for the proof of our next theorem, we use the fact that if $A$ is in $\mathscr{L}(E)$ and $w-\lim _{n \rightarrow \infty} x_{n}=x$, then $w-\lim _{n \rightarrow \infty} A x_{n}=A x$.

THEOREM 2. Suppose that $W$ is defined by (3), $M$ is defined by (5), and $W$ is asymptotically convergent. In addition, suppose that there is an $x_{0}$ in $E$ and a sequence $\left(t_{n}\right)_{1}^{\infty}$ in $S$ such that $\lim _{n \rightarrow \infty} t_{n}=\infty$ and $w$ - $\lim _{n \rightarrow \infty} M\left(0, t_{n}\right) x_{0}$ exists. Then $z$ is in the range of $A$ and the conclusions of Theorem 1 are valid.

Proof. Using (7) and the fact that $Q W(s, t)=Q$ (see (ii) of Lemma 2), we have that

$$
\begin{aligned}
Q M\left(0, t_{n}\right) x_{0} & =Q W\left(0, t_{n}\right) x_{0}+Q(R) \int_{0}^{t_{n}} W\left(\cdot, t_{n}\right) z d g \\
& =Q x_{0}+(R) \int_{0}^{t_{n}} Q z d g=Q x_{0}+g\left(t_{n}\right) Q z .
\end{aligned}
$$

Thus $Q z=\theta$ since $\lim _{n \rightarrow \infty} g\left(t_{n}\right)=\infty$. Again using (7),

$$
\begin{aligned}
A M\left(0, t_{n}\right) x_{0} & =A W\left(0, t_{n}\right) x_{0}+A(R) \int_{0}^{t_{n}} W\left(\cdot, t_{n}\right) z d g \\
& =A W\left(0, t_{n}\right) x_{0}+(R) \int_{0}^{t_{n}} A W\left(\cdot, t_{n}\right) z d g \\
& =A W\left(0, t_{n}\right) x_{0}+W\left(0, t_{n}\right) z-z,
\end{aligned}
$$

where (4) was employed to obtain the last identity. Hence, if $x^{*}=$ $w-\lim _{n \rightarrow \infty} M\left(0, t_{n}\right) x_{0}$,

$$
A\left(-x^{*}\right)=-w-\lim _{n \rightarrow \infty} A M\left(0, t_{n}\right) x_{0}=-A Q x_{0}-Q z+z=z,
$$

and the assertions of Theorem 2 follow.

As an example of the above results, let $S$ be the set of nonnegative integers, let $\left(\lambda_{k}\right)_{1}^{\infty}$ be a sequence of positive numbers such that $\sum_{1}^{\infty} \lambda_{k}=\infty$, and let $g(n)=\sum_{1}^{n} \lambda_{k}$ for each $n \in S$ (where $\sum_{1}^{0} \lambda_{k}=0$ ). If we let $A=T-I$, then it is easy to see that

and

$$
W(0, n) x=\prod_{1}^{n}\left[\left(1-\lambda_{k}\right) I+\lambda_{k} T\right] x,
$$

$$
M(0, n) x=\prod_{1}^{n}\left[\left(1-\lambda_{k}\right) I+\lambda_{k}(T+z)\right] x
$$

for each $n \in S$ and $x \in E$. In particular, $W$ and $M$ satisfy the recursion 
formulas

and

$$
W(0, n+1) x=\left(1-\lambda_{n+1}\right) W(0, n) x+\lambda_{n+1} T W(0, n) x,
$$

$$
M(0, n+1) x=\left(1-\lambda_{n+1}\right) M(0, n) x+\lambda_{n+1} T M(0, n) x+\lambda_{n+1} z
$$

for each $n \in S$. If there is a number $\lambda$ in $(0,1]$ such that $\lambda_{k}=\lambda$ for all $k \geqq 1$, we have that

and

$$
W(0, n) x=[(1-\lambda) I+\lambda T]^{n} x,
$$

$$
M(0, n) x=[(1-\lambda) I+\lambda(T+z)]^{n} x
$$

for each $n \in S$ and $x \in E$. The variation of parameter formula (7) shows also that $M(0, n+1) x=[(1-\lambda) I+\lambda T]^{n+1} x+\sum_{0}^{n}[(1-\lambda) I+\lambda T]^{k} z$. In particular, setting $\lambda=1$, we have the result of Browder and Petryshyn [1]. If $S=[0, \infty)$ and $g(t)=t$ for all $t \in S$, then $W(s, t)=\exp ((t-s) A)$ for all $(s, t) \in \Delta$ and $M(s, \cdot) x$ is the solution $u$ to the differential equation $u^{\prime}(t)=A u(t)+z$ which satisfies $u(s)=x$. In this case (7) is the classical variation of parameters formula.

In closing, let us point out that a theory involving the mean asymptotic convergence of $W$ can also be developed using Stieltjes integral equations. Define the function $P$ from $S$ into $\mathscr{L}(E)$ by $P(0)=I$ and, for each $t \in S$, $t \neq 0$, let

$$
P(t) x=g(t)^{-1}(L) \int_{0}^{t} W(0, \cdot) x d g
$$

for all $x \in E$. Then $W$ is said to be mean asymptotically convergent if $Q x=\lim _{t \rightarrow \infty} P(t) x$ exists for each $x \in E$. Using techniques similar to the above, one can establish theorems on mean convergence analogous to Theorems 1 and 2 . However, this type of theory is subsumed directly by the results of Dotson [3], [4].

\section{REFERENCES}

1. F. E. Browder and W. V. Petryshyn, The solution by iteration of linear functional equations in Banach spaces, Bull. Amer. Math. Soc. 72 (1966), 566-570. MR 32 \#8155a.

2. D. G. DeFigueiredo and L. A. Karlovitz, On the approximate solution of linear functional equations in Banach spaces, J. Math. Anal. Appl. 24 (1968), 654-664. MR 38 \#6415.

3. W. G. Dotson, Jr., An application of ergodic theory to the solution of linear functional equations in Banach spaces, Bull. Amer. Math. Soc. 75 (1969), 347-352. MR 39 \#795.

4. - Mean ergodic theorems and iterative solution of linear functional equations, J. Math. Anal. Appl. 34 (1971), 141-150. 
5. D. L. Lovelady, Perturbations of solutions of Stieltjes integral equations, Trans. Amer. Math. Soc. 155 (1971), 158-178.

6. J. S. Mac Nerney, Integral equations and semigroups, Illinois J. Math. 7 (1963), 148-173. MR 26 \#1726.

7. $\longrightarrow, A$ nonlinear integral operation, Illinois J. Math. 8 (1964), 621-638. MR 29 \#5082.

8. J. A. Reneke, $A$ variation of parameters formula (unpublished manuscript).

Department of Mathematics, North Carolina State University, Raleigh, North Carolina 27607

Current address: Mathematics Division, University of Sussex, Falmer, Brighton, England 